\title{
Sustained Mnemonic Response in the Human Middle Frontal Gyrus during On-Line Storage of Spatial Memoranda
}

\author{
H.-C. Leung, J. C. Gore, and P. S. Goldman-Rakic
}

\begin{abstract}
- The mapping of cognitive functions to neural systems is a central goal of cognitive neuroscience. On the basis of homology with lesion and physiological studies in nonhuman primates, Brodmann's area (BA) 46/9 in the middle frontal gyrus (MFG) has been proposed as the cortical focus for both the storage as well as processing components of working memory in the human brain, but the evidence on the segregation of these components and their exact areal localization has been inconsistent. In order to study this issue and increase the temporal resolution of functional mapping, we disambiguated the storage component of working
\end{abstract}

\section{INTRODUCTION}

Working memory is widely considered to consist of a storage component in which information is accessible in an activated state and a processing component for manipulating that information in the execution of cognitive functions (Miyake \& Shah, 1999; Baddeley, 1986). Insight into the neural machinery of working memory has come from lesion studies and from single-unit recordings in the dorsolateral prefrontal cortex of nonhuman primates (Goldman-Rakic, 1987). Lesions of this area (Brodmann's area 9; Walker's area 46) result in profound and longlasting deficits in performance on spatial delayedresponse (DR) tasks (Goldman, Rosvold, Vest, \& Galkin, 1971), and prefrontal neurons within this area exhibit persistent activity during delay periods distinct from neuronal responses to sensory and motor events (Funahashi, Bruce, \& Goldman-Rakic, 1989; Niki, 1974a; Fuster \& Alexander, 1971). These and other findings have served as the basis for proposing that Brodmann's area (BA) 46 or $46 / 9$ in the human brain might also be preferentially engaged in "on-line" storage as well as in the processing of spatial information and, by extension, that cognitive architecture is conserved in the human and nonhuman brain as it is for sensory and motor systems. While many functional magnetic resonance imaging (fMRI) studies have supported a prominent role of the human dorso-

Yale University memory from sensory and motor responses by employing functional magnetic resonance imaging (fMRI) in spatial delayed-response (DR) tasks with long delay intervals and different conditions of demand. We here show that BA 46 can support a sustained mnemonic response for as long as $24 \mathrm{sec}$ in a high-demand task and the signal change in this area exceeded that in the other prefrontal areas examined. Our findings support a conservation of functional architecture between human and nonhuman primate in showing that the MFG is prominently engaged in the storage of spatial information.

lateral prefrontal cortex (BA 46/9) in spatial processes (Zarahn, Aguirre, \& D'Esposito, 1999, 2000; Belger et al., 1998; Carlson et al., 1998; Goldberg, Berman, Randolph, Gold, \& Weinberger, 1996; McCarthy et al., 1996; Sweeney et al., 1996; O'Sullivan, Jenkins, Henderson, Kennard, \& Brooks, 1995), others have reported preferential activations at other sites, including the premotor (PM, BA 6/8) (Rowe, Toni, Josephs, Frackowiak, \& Passingham, 2000; Zarahn et al., 1999; Courtney, Petit, Maisog, Ungerleider, \& Haxby, 1998) and ventrolateral (BA 44/45/47) (Owen, Evans, \& Petrides, 1996; Smith, Jonides, \& Koeppe, 1996; Smith et al., 1995; Jonides et al., 1993) prefrontal cortex. Furthermore, findings from a variety of studies using complex cognitive tasks have been taken as support for a hierarchical organization of prefrontal cortex by which higher order functions (e.g., manipulation) are allocated to dorsolateral prefrontal areas and the simpler maintenance components assigned to inferior prefrontal areas (Owen et al., 1999; D'Esposito et al., 1998; Petrides, 1994a). Thus, the role of BA 46 and 9 in the storage component of spatial working memory is in question, and beyond that, the localization and segregation of component storage and/or processing functions in the human frontal lobe remain unresolved.

We reason here that disambiguating the mnemonic response in spatial delay tasks from sensory and motor activations may be helpful, if not essential, in establishing accurate functional maps of elemental cognitive processes in the prefrontal cortex. In particular, the 
relatively short retention intervals $(3.5-12 \mathrm{sec})$ used in many previous fMRI studies of spatial working memory tasks (e.g., Zarahn et al., 1999; Belger et al., 1998; Courtney et al., 1998) may have measured a merged sensorimotor signal rather than activations related to the mnemonic process per se. A higher resolution of regional distribution in relation to task events may result from the use of an extended delay period.

Accordingly, in order to isolate the mnemonic component of spatial working memory in the human brain, as well as more accurately resolve the temporal dynamics of this process, we have examined cortical activations with fMRI as subjects maintained visuospatial memoranda "on-line" over 18- and 24-sec delay periods. This approach allowed us to directly measure the mnemonic component in the fMRI signal and reveal regional differences in the strength, duration, and sequence of activation as a function of memory load.

\section{RESULTS}

Our findings are based on the results from 30 subjects in three independent experiments all using a DR format with long delays but differing in sensory and/or memory requirements (Figure 1A). In Experiment 1, subjects had to keep in mind the location of five stimuli (dots) presented sequentially over $18 \mathrm{sec}$. The number of memoranda for this experiment was decided upon after failing to observe prefrontal activity with only one or two items in a pilot study (Leung, Gore, \& Goldman-Rakic, 1999). The sensorimotor control condition was identical except that subjects were instructed not to remember the stimuli. At the end of the 18-sec delay, a single stimulus reappeared as a probe and the subject had to indicate whether the location indicated by the dot had been in the original list. Thus, the correct response in the experimental condition was guided by memory; whereas in the control condition, it was sensory guided. Experiment 2 examined the effect of delay duration by comparing behavioral performance and cortical activation in the same five-item task with delay periods of 18 and $24 \mathrm{sec}$, respectively. Experiment 3 examined load effects by comparing performance and event-related activations in a three-item (DR-3) task with that in a five-item (DR-5) task. In Experiment 3, as expected, the level of behavioral performance varied with the number of items to be recalled. This was observed in the reaction time data, which increased from 1271 to $1681 \mathrm{msec}$ in the DR-3 and DR-5 tasks, respectively, $t(20)=2.09$, $p<.02$. Accuracy measures $(94 \%$ and $78 \%$ for DR-3 and DR-5, respectively) also reflected a significant task effect, $t(20)=2.09, p<.001$. Comparisons of experimental versus control reaction time data in the DR-3 (1271 vs. $958 \mathrm{msec}$ ) and DR-5 (1681 vs. $1012 \mathrm{msec}$ ) tasks were also highly significant $(p<.0005)$.

Increases in activation from fixation baseline were seen in all task events—cue, delay, and response in all experi-
A. Delayed-response spatial working memory tasks Exp. 1 Memory vs. control

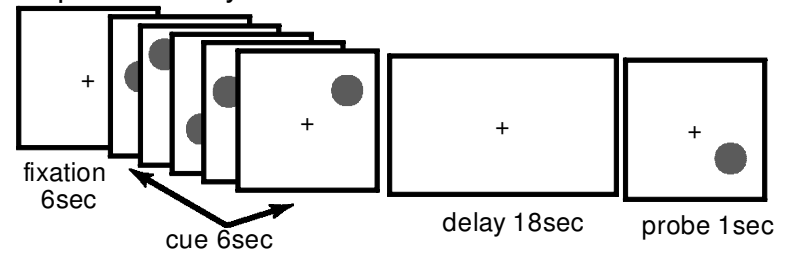

Exp. 2 Variable delay duration (18 vs. 24sec)

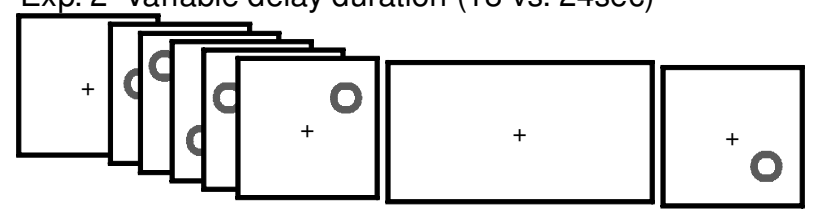

Exp. 3 Memory load (3 vs. 5 items)

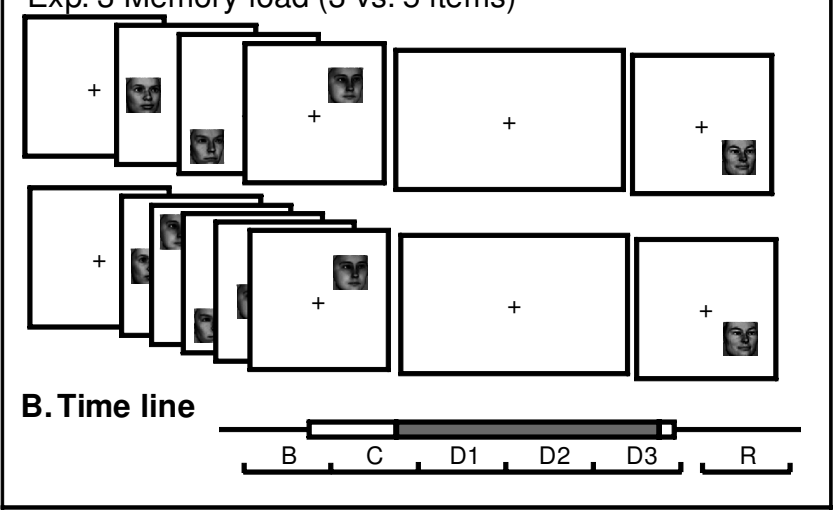

Figure 1. Schematic diagram of spatial working memory task events. (A) Sequential trial events in the DR tasks. Experiment 1: The memory condition of a five-item task. The no-memory condition (sensorimotor control, not shown) has the same sequence of events, except subjects were instructed not to remember the target locations. Experiment 2: Five-item task with a retention interval of $24 \mathrm{sec}$. This condition was compared with an 18-sec delay condition (not shown). Experiment 3: Memory conditions for three- and five-item tasks. Note that the 18-sec delay interval was the same for tasks in both Experiments 1 and 3. (B) The bottom drawing shows how images were grouped for activation analyses across epochs corresponding to: cue (C), delay (D1, D2, D3), and response $(\mathrm{R})$. Each group was comprised of four images (6 sec). Baseline (B) images included three images before and one after cue onset. Statistical maps were generated by calculating the percent signal change from baseline in each interval. Open bars = visual stimulus duration; gray bar = delay duration; lines $=$ fixation and ITI periods

ments. The 18-sec delay period was divided into three 6-sec periods (Figure 1B): D1 (cue-delay period), D2 (mid-delay), and D3 (late delay). As a rule, higher signals were observed during the D1/D2 and response (R) periods than during the later delay intervals and similar patterns of activations were observed across experiments.

\section{Experiment 1: Isolation of the Memory Component (Memory vs. Control in a Five-Item Spatial Delayed Response Task)}

Activations above fixation baseline for the memory condition were observed in the left precentral sulcal (PrCS) and premotor (PM) cortices, the right MFG and 


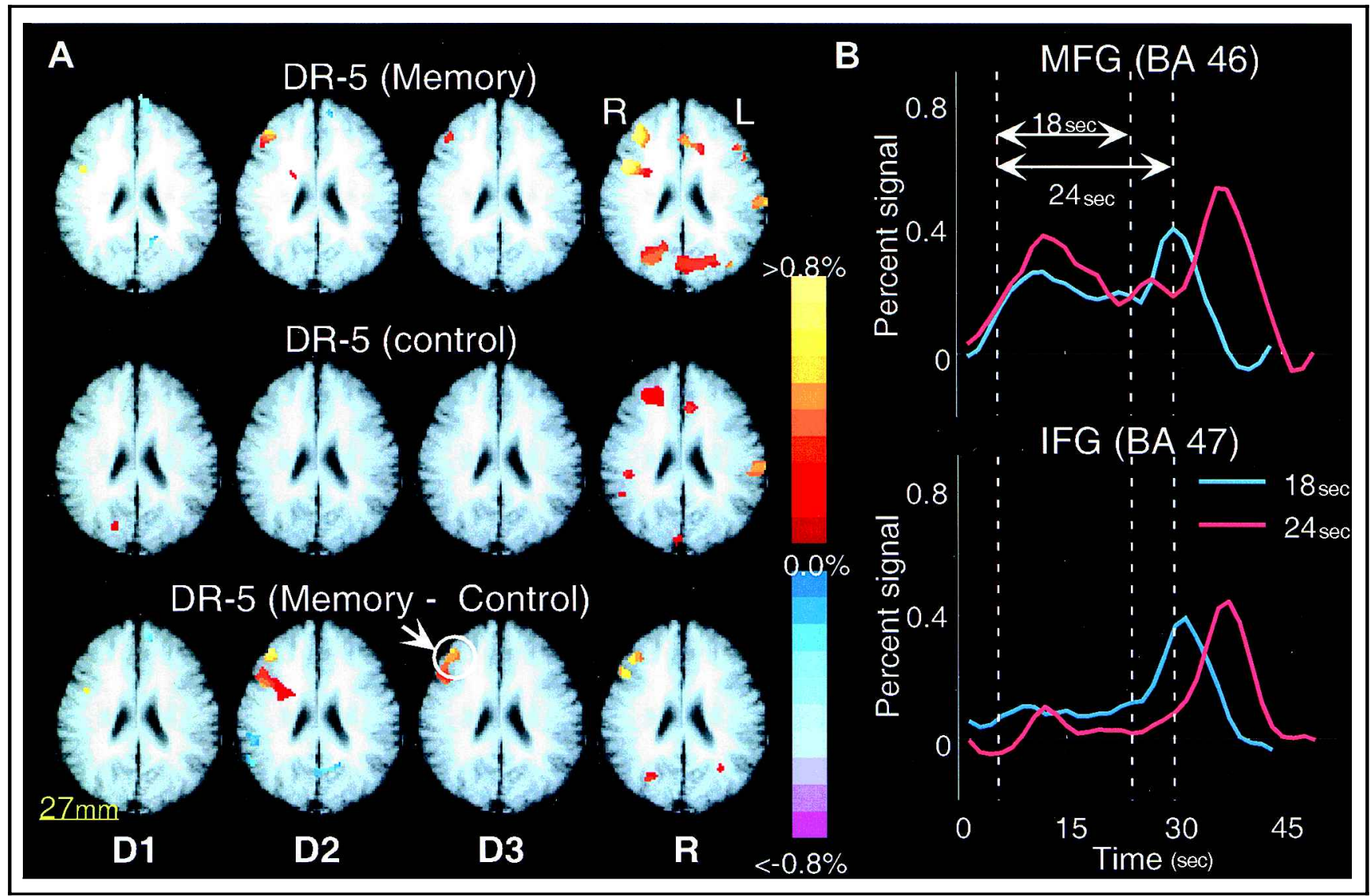

Figure 2. Results from Experiments 1 and 2 showing sustained activation in the MFG. (A) Each row shows activation maps of a slice include MFG (BA 46, $z=27 \mathrm{~mm}$ ) across time blocks (D1, D2, D3, and R): from top to the bottom: DR-5 memory condition, DR-5 no-memory control, and subtraction between the two. Individual maps represent average percent signal changes during the three segments of delay and response epochs relative to the fixation baseline. Persistent activation through the entire delay was found only in the memory condition. The mean percent signal changes are shown only for areas that were activated above a $p<.005$ threshold (uncorrected, see methods). BA 46 is indicated by a white circle and arrow. Positive activations are indicated by red to yellow, and negative activations by blue to purple. $\mathrm{R}=$ right; $\mathrm{L}=1$ left. (B) Average percent signal changes from fixation baseline are plotted for the DR-5 task at two delays (18 sec/blue line and 24 sec/red line). Note that the enhanced activity in the MFG (BA 46) remained elevated after $18 \mathrm{sec}$ in the longer delay condition. However, sustained activity was not observed in the IFG BA 47. The first vertical line marks the beginning of the delay period and the second and third lines mark the onset of the response probe presentation for the 18- and 24-sec delay conditions, respectively. See Figure 1 for notations and Table 1 for abbreviations.

bilaterally in the inferior frontal gyrus (IFG), the parietal/ occipital junction, and lateral occipital areas. Outside of the prefrontal cortex, long-lasting activations were present only in the superior parietal (SPL), PrCS, and PM areas. Within the prefrontal cortex, the most prominent signal was observed in the right middle frontal gyrus (MFG) and this signal remained suprathreshold until the end of the delay period (Fig. 2A, $p<0.005$, uncorrected). The activation in BA46 extended though the last segment (D3) of the delay period and remained significantly higher than that of the control condition in this segment ( $p<0.05$, Fig. $2 \mathrm{~A}$, bottom row).

\section{Experiment 2: Durability of Delay-Related Activations (18 vs. 24 sec of Retention Interval)}

As previous single-unit studies in nonhuman primates have demonstrated that the persistent activity of pre- frontal neurons can be extended to accommodate longer delays (Funahashi et al., 1989; Niki, 1974a; Fuster \& Alexander, 1971), we examined whether a similar flexibility can be observed in the human fMRI signal. We therefore compared the duration of the hemodynamic response in both 18 - and $24-\mathrm{sec}$ retention intervals in four additional subjects. Performance measures in the 18- and 24-sec conditions did not differ significantly (reaction time: 1477 vs. $1621 \mathrm{msec}$, $p>.05$; accuracy: $73 \%$ vs. $69 \%, p>.05$ ) and a sustained hemodynamic signal in the MFG was observed throughout the longer 24-sec interval as in the 18-sec delay (Figure 2B, top). Paralleling results in the nonhuman primate, these findings indicate that the hemodynamic response elicited by a storage task in humans is robust and durable as required by the temporal dimensions of the task, at least up to the maximum time employed in this study. Similar 
Figure 3. Percent signal difference between the memory and control conditions of DR-3 and DR-5 tasks (Experiment 3). These group composite maps show activations based on contrasts between the memory and control conditions of (A) DR-3, (B) DR-5. Mean percent signal differences for areas above a threshold of $p<.005$ (uncorrected) are shown across five time blocks (in columns). Note that activations during the memory condition of DR- 5 are greater than that of the control for multiple frontal and parietal areas and are persistent throughout the entire delay interval (D1-D3) for these areas. There is little MFG activation in the DR-3 task and it did not last. The slice with BA 46 is highlighted $(z=27 \mathrm{~mm})$ and the MFG is indicated by white circles and arrows. See Figures 1 and 2 for notations.
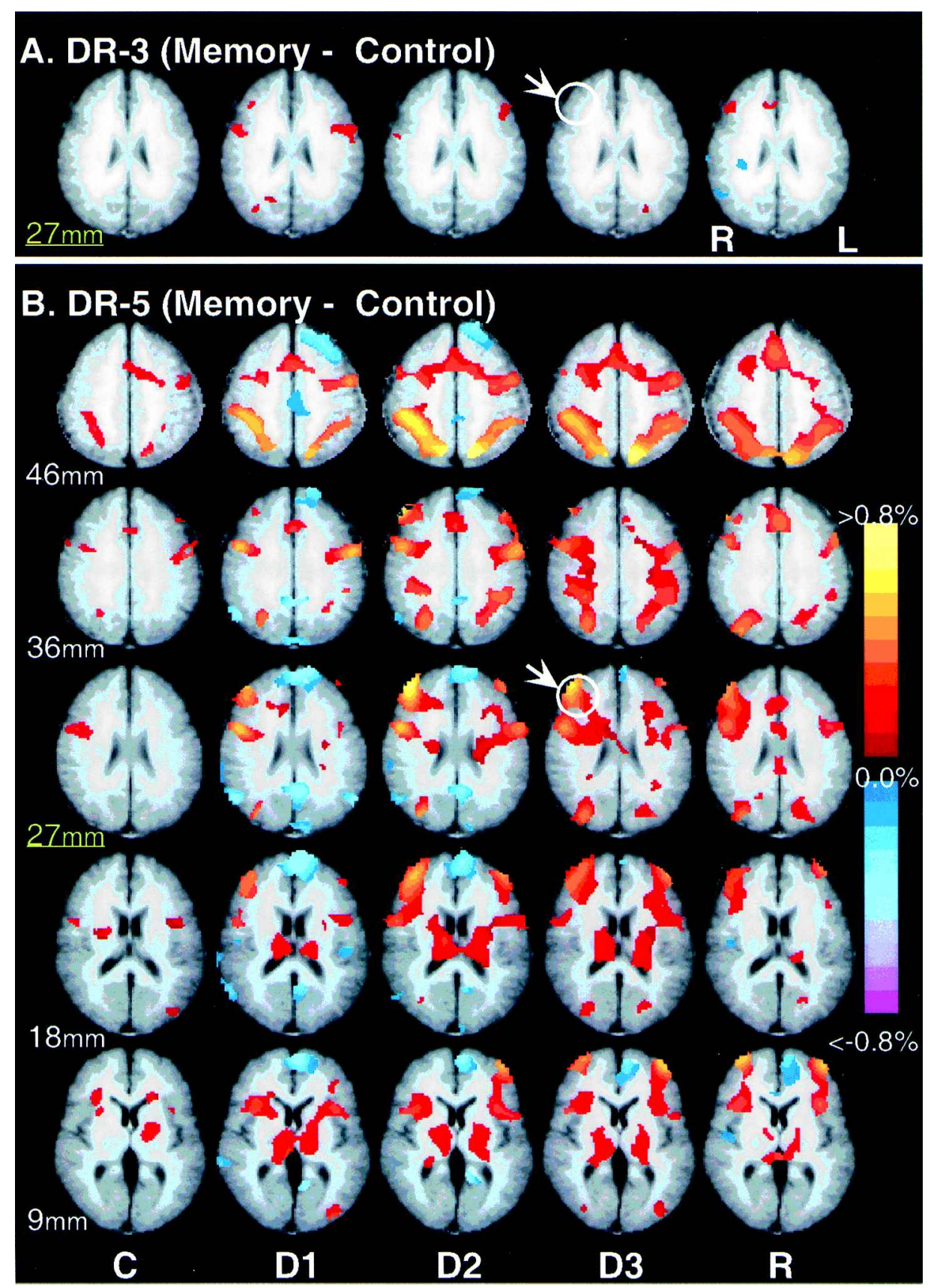

sustained activity was not observed in the IFG (BA 47, Figure 2B, bottom).

\section{Experiment 3: Memory Load Effects}

The robust storage-related MFG activations observed in Experiments 1 and 2 contrast with negative or weak activations in this area reported in several recent imaging studies of spatial working memory with three-item recall (Rowe et al., 2000; Courtney et al., 1998). To test the hypothesis that the difference in results across studies might be due to differences in memory load, we compared three- and five-item formats using the same task design and 18-sec delay period as in Experiments 1 and 2, but used face stimuli for direct comparison with Courtney et al. (1998). In the DR-3 task of the present series, we observed suprathreshold activations above fixation baseline mainly during the D1, D2, and $\mathrm{R}$ intervals in the PrCS, the inferior parietal lobe (IPL), the lateral occipital areas, and prefrontal areas including the MFG and IFG. The anterior cingulate (ACG) was activated primarily during the response period. Although significant percent signal differences were observed during the delay period when comparing 
memory versus control conditions in the MFG (BA 46), PrCS (BA 6/44), and IPL (BA 40) (repeated measure, $p<.05$, corrected), none of these regions of interest (ROIs) showed significant activations during the D3 segment of the delay period (Figures 3A and 4).

As expected, the same regions that were activated in DR-3 were also activated in DR-5. Multiple areas in the prefrontal, PM, and parietal cortices all showed significant increases in signal during the DR-5 memory condition relative to the control condition (Figure $3 \mathrm{~B}$ and Table 1). In contrast to DR-3, the DR-5 memory condition also elicited the most persistent delay-related activation. It is notable that the strongest percent increase in signal in the prefrontal cortex relative to control values was observed in the MFG in all delay segments including the D3 interval. Moreover, the suprathreshold D3 signals in the MFG were localized in ROIs centered within or on the posterior/ventral border of BA 46, adjacent to BA 9 and 10 (Table 1, top rows). The Talairach coordinates of these ROIs were within or on the border of BA 46 defined by cytoarchitectonic criteria (Rajkowska \& Goldman-Rakic, 1995). Within this zone, the strongest signal change was observed in the ventral bank of the superior frontal sulcus (SFS) (the average center of mass at the Talairach coordinates: 33, 44, 27). Notably, prefrontal ROIs cen- tered in BA 6/8, 9, 10, 44, and 24/32 were not significantly activated in the $\mathrm{D} 3$ period.

To further evaluate the time-dependence of activations, average percent signal change from baseline was plotted over time for ROIs in both tasks (Figure 4, insets). Most activations peaked during D1 and D2 and decayed substantially by $\mathrm{D} 3$ before reemerging at the time of the response probe. In the DR-3 task, activity decayed to near baseline after peaking around $6 \mathrm{sec}$ following cue offset, whereas in the DR- 5 task, signals peaked around $8 \mathrm{sec}$. In the latter task, signals in MFG, IFG, PM, PrCS, and SPL decayed during memory trials but nonetheless remained on average above baseline and control task levels throughout the entire 18-sec delay period. Further, the results from this DR-5 experiment not only replicate the MFG findings from Experiment 1 , but reveal a high degree of reliability, as those subjects that participated in both experiments (conducted at different times and with different stimulus conditions) showed the same heightened MFG activations during the late segment (D3) of the long delay interval in both DR-5 tasks (Figure 5).

In addition to within-task time-course analysis, we also compared the timing of peak activations in DR-5 across regions and found a posterior to anterior progression of areal involvement as well as significantly later
Figure 4. Average percent signal changes and temporal dynamic differences between tasks. Average percent signal changes for four ROIs are shown in bar charts. Key: white $=$ DR-3 control condition (3C); hatch = DR-3 memory condition $(3 \mathrm{~T})$; gray $=$ DR-5 control condition $(5 \mathrm{C})$; black $=$ DR-5 memory condition (5T). Standard error bars are shown in the plots. The inset of each panel shows time courses of DR-3 (dashed line, 3T) and DR-5 (solid line, 5T). The $x$-axis represents time and the $y$-axis represents average percent signal change from fixation baseline. Time zero represents the onset of the first cue. Vertical lines in each panel mark the beginning and end of the delay period $(18 \mathrm{sec})$. BA are shown in parentheses. Note that signal changes during DR-5 are consistently higher than that during DR-3, with the exception of the visual area. See Figure 1 for notations and Table 1 for abbreviations.

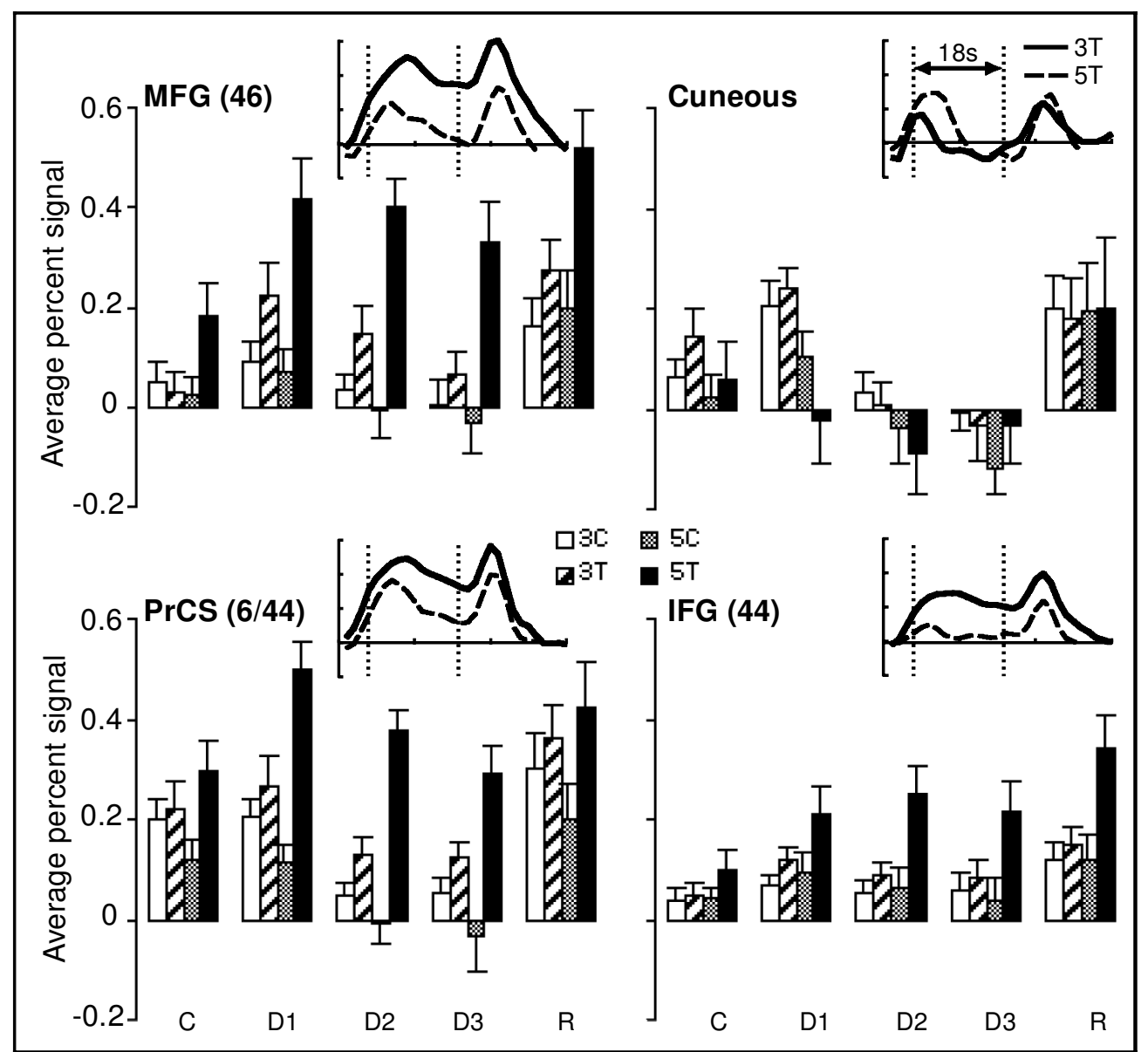




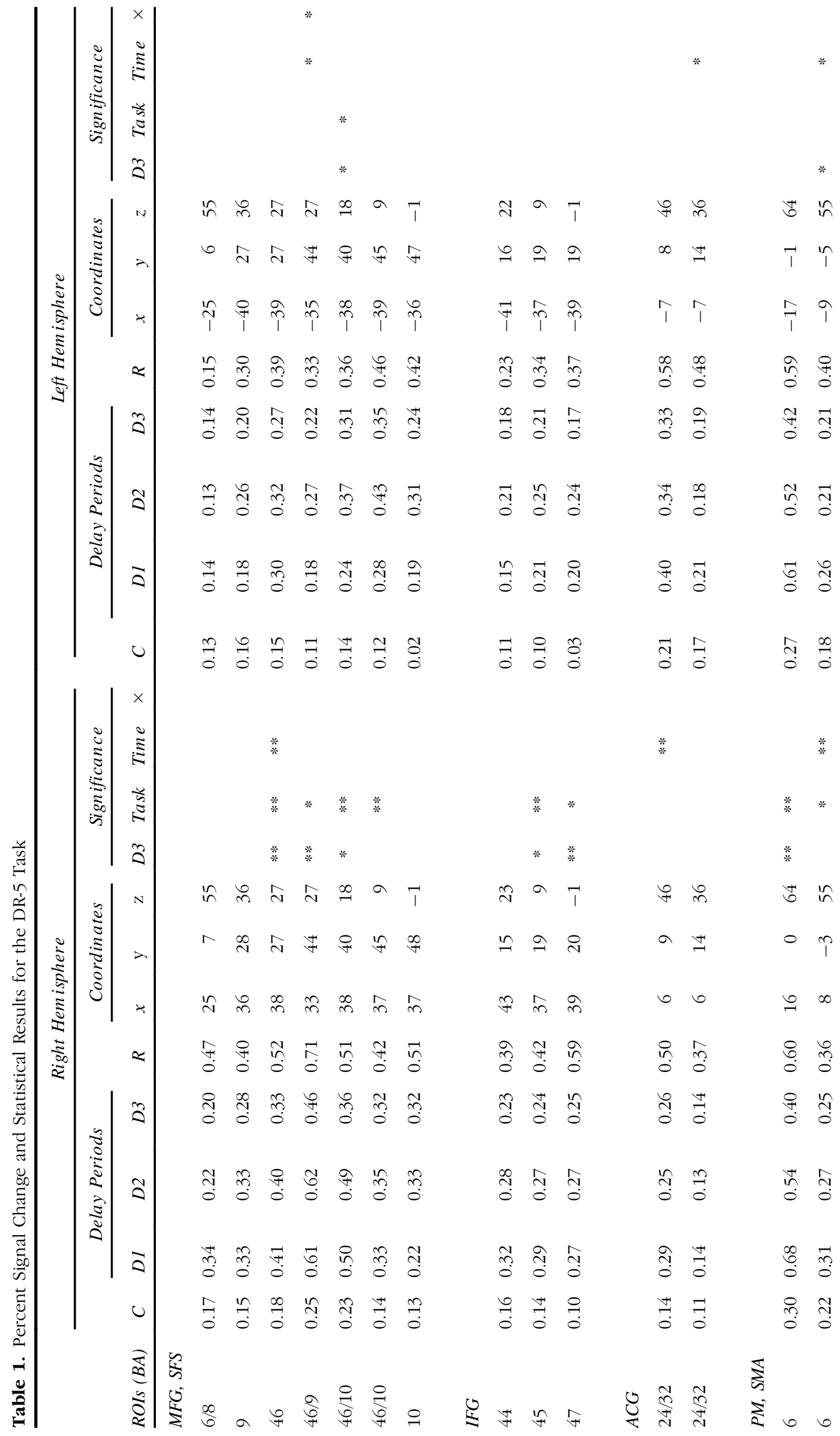




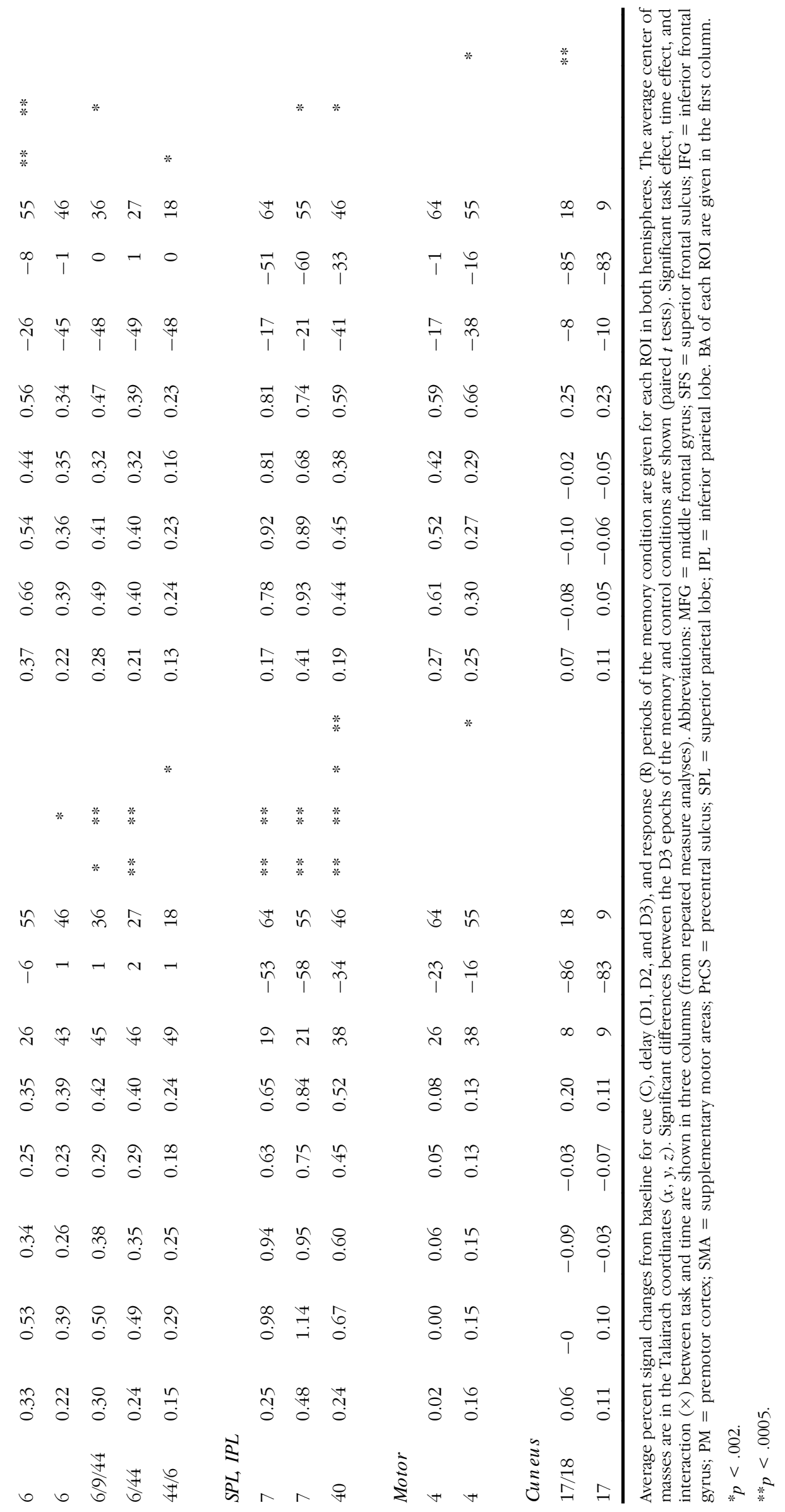



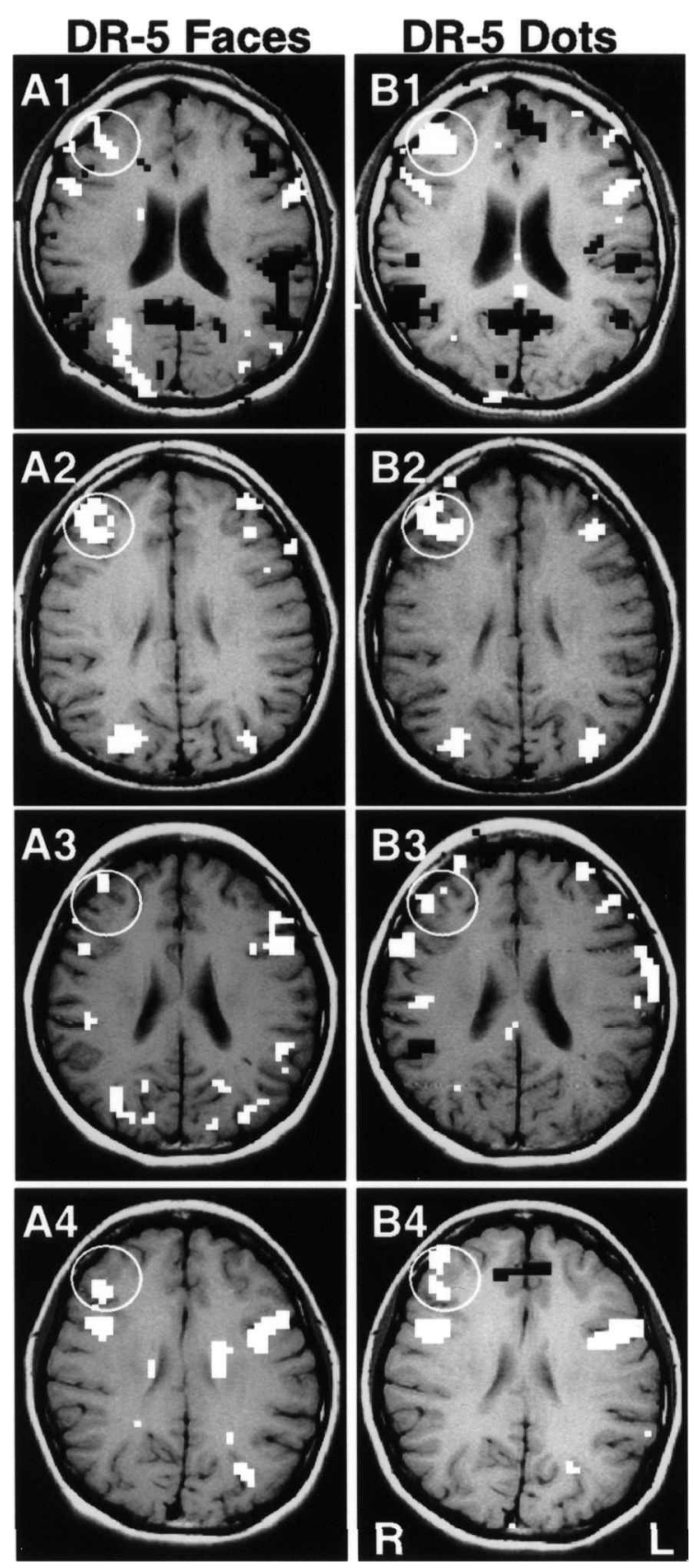

Figure 5. Pattern of activations in individual subjects for both DR-5 tasks. Activation maps shown are obtained by contrasting the last delay segments (D3) of memory and control conditions in four subjects that participated in both DR-5 tasks using faces (A1-A4) and dots (B1-B4) as stimuli. Only pixels that are within the top $2.5 \%$ of total activation of the slice are shown (positive and negative signals are in white and black, respectively). Note that MFG activations (marked by white circles) are conserved in both DR-5 tasks against their own control. See Figures 1 and 2 for notations.

peak activity of activated regions during the memory condition than during the control condition, $F(1,20)=$ $13.228, p=.0016$; Figure 6 . The earliest peak activity was observed at the end of the cue period in the occipital lobe (BA 17/18). Responses in PM, SPL, and
PrCS peaked next, between 4.5 and 12 sec after cue offset, and earlier than those in the IFG and MFG. An important observation for consideration in designing studies of MFG function is that the hemodynamic signal in the composite MFG region (BA 46/10) was the last to peak among the ROIs examined (Figure 6), including the PrCS, SPL, and PM. Thus, paradigms using short delays could miss this peak in the MFG. In contrast to the temporal sequence of activations in the delay interval, peak activations occurred concurrently in all ROIs at the time of the response probe presentation and did not differ between the memory and control conditions $(p>.05)$, further indicating that it is the delay (i.e., the storage period) that distinguishes the function of these areas during spatial working memory performance.

\section{DISCUSSION}

The role of the prefrontal cortex in cognitive function has been a subject of current interest and much debate (Duncan \& Owen, 2000; Smith \& Jonides, 1999; D'Esposito et al., 1998; Owen, 1997). One prominent view initially proposed by Petrides is that the dorsolateral is selectively engaged in the updating and manipulation of information, while the maintenance function of working memory is reserved for ventral prefrontal areas, irrespective of the content of information (Owen et al., 1999; Petrides, 1994a, 1994b). This view therefore suggests a segregation of storage and processing functions, whereas findings from studies of nonhuman primates in this laboratory suggest that these functions may be domain specific, integrally related, and carried out by the same intrinsic circuitry (for review, see Goldman-Rakic, 1996). Since the role of the dorsolateral prefrontal cortex in on-line processing of spatial information is not in contention (D'Esposito, Postle, Ballard, \& Lease, 1999; Levy \& Goldman-Rakic, 1999; Postle, Berger, \& D'Esposito, 1999; Goldman-Rakic, 1987), the present study was designed to examine whether it is also engaged during storage- - a function more akin to the slave systems of working memory (Baddeley, 1986). If it is, then storage and processing of one cardinal knowledge domain-spatial location-would appear to be integrated within a circumscribed cortical area and not segregated to different areas.

\section{Maintenance Function of the MFG}

The DR format used in the present study was selected specifically to avoid or minimize processing requirements beyond short-term storage of information. Because subjects were shown a sequence of stimuli marking different locations but did not know which location would be relevant until the end of the delay, activations during the delay period could not be argued to represent a preparatory motor response or verbal rehearsal. Neither is it reasonable to attribute the sustained activation observed 
Figure 6. Posterior-anterior progression of peak responses for DR-5. (A) Time of the first peak, after cue presentation. The arrow marks the end of cue presentation, which is the beginning of the delay interval (B) Time of the second peak, after the response probe (mark by an arrow). The $x$-axes represent time from the beginning of cue presentation. Standard error bars are shown in each plot. *Significant difference in peak time between the three areas and the visual area. Key: gray $=$ memory condition $(5 \mathrm{~T})$; white $=$ control condition $(5 \mathrm{C})$.

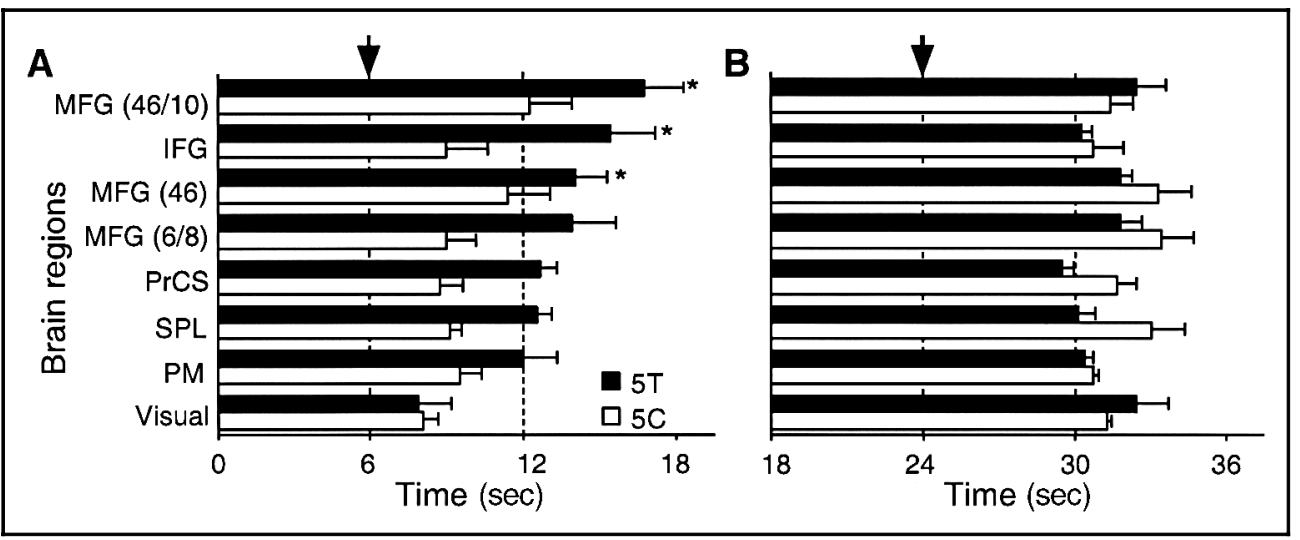

in DR-5 to a prolonged sensory response. First, the signal change observed to the stimuli did not last throughout the delay in the three-item memory condition, and resolved in the D1 segment of the delay in all control conditions. Further, the similar findings obtained with simple geometric stimuli and faces provided further evidence that delay-related activation is independent of the nature of visual stimuli.

It could be argued that subjects employed processing strategies during the five-item (higher demand) tasks that accounted for the longer duration activations observed in these tasks. Although it is difficult to rule out

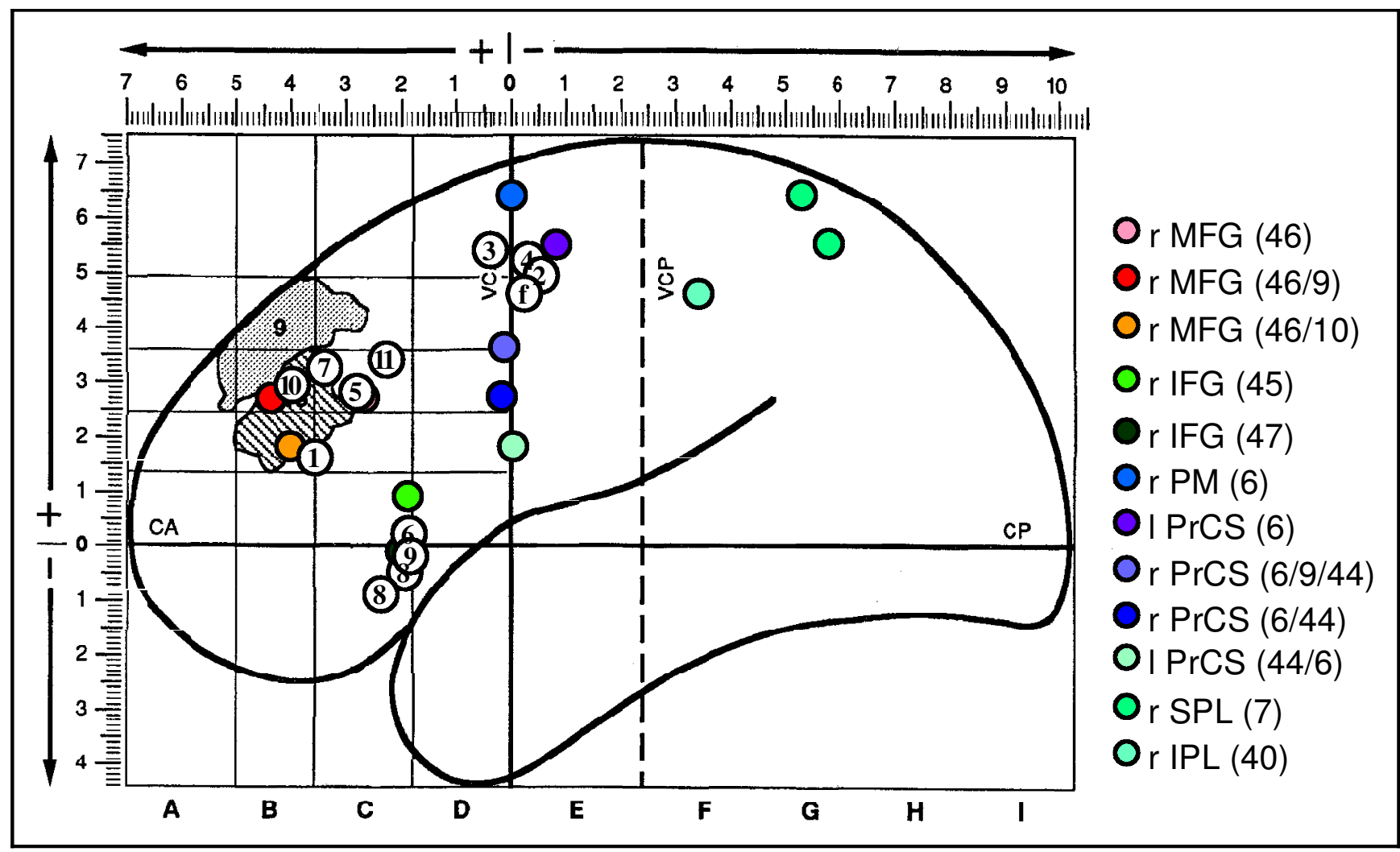

Figure 7. Center of masses for DR-5 activations. The average center of masses for significant D3 activations within the ROIs are plotted in the Talairach coordinate system. The schematic diagram of the left lateral brain was from Rajkowska and Goldman-Rakic (1995), which shows the common regions of overlap in cytoarchitectonic reconstructions of BA 9 and 46 from five human brains. Note most of the loci from the current study are in the right hemisphere (see Table 1 for notations and exact coordinates). All the significantly activated areas in the MFG are centered within or near the previously conservatively defined BA 46. Numbered circles mark the coordinates for prefrontal activations from selected DR $(1-8)$ and other $(9-11)$ spatial working memory studies (see References). The functional localization of frontal eye field (FEF) reviewed by Paus (1996) is also indicated (f). BA are shown in parentheses ( $\mathrm{r}=$ right; $1=$ left). Key: $1=$ Belger et al. (1998); $2=$ Courtney et al. (1998); $3=$ Rowe et al. (2000); $4=$ Zarahn et al. (1999); $5=$ Sweeney et al. (1996); $6=$ Jonides et al. (1993); $7=$ O'Sullivan et al. (1995); $8=$ Owen et al. (1996); $9=$ Smith et al. (1996); $10=$ McCarthy et al. (1996); $11=$ D'Esposito et al. (1998). 
any processing in simple DR tasks, it is also difficult to envision a strategy other than visuospatial representation that could be implemented in these tasks, and indeed, self-reports of the subjects indicated that they did not consciously employ verbal or other strategies for either the three- or five-item tasks. This is entirely reasonable as the human memory span for spatial location has been estimated to be approximately five items or more (Jiang, Olson, \& Chun, 2000; Smyth \& Scholey, 1992). If the ventral prefrontal cortex were selectively dedicated to storage functions independent of domain, we should have observed greater or equally strong activation in the IFG than in the MFG. The persistent MFG activations observed here instead support an "on-line" storage function for this region, consistent with an economy of brain circuitry for storage and processing of information in the same circuits.

\section{Storage Threshold for Activation of the MFG}

The robust storage-related MFG activation observed in the present study contrasts with negative or weak activations in this area reported in several recent imaging studies. Rather, as stronger signals were observed in the IFG (Smith et al., 1995, 1996; Jonides et al., 1993), PrCS (Zarahn et al., 1999; Courtney et al., 1998), or PM (Rowe et al., 2000) in these studies, the on-line storage function was attributed to one or more of these sites, as opposed to the MFG. The load effects observed here may explain this discrepancy. We observed strong and lasting activation in the MFG only with a five-item memory requirement, and thus insufficient memory demand is likely the reason for why simple three-item storage tasks, employed in the present and in previous studies, failed to engage prolonged activation in this area. By this account, BA 46 in the MFG of the dorsolateral prefrontal cortex would be placed in a hierarchical functional relationship to other areas, as the clinical literature dictates, and the IFG, PrCS, PM, and posterior association areas (e.g., parietal cortex) would be sufficient to subserve tasks with lower storage demands. Load effects in dorsolateral prefrontal cortex have previously been reported for verbal material in DR-formatted tasks (Rypma, Prabhakaran, Desmond, Glover, \& Gabrieli, 1999). Similar conclusions have been reached in other studies using $n$-back designs that require constant updating of verbal (Cohen et al., 1997) and spatial (Carlson et al., 1998) memoranda. The present findings thus extend the evidence for the impact of load to spatial DR tasks, and as well, to the duration of the mnemonic response.

\section{Conservation of Functional Architecture in Evolution: Parallels With Nonhuman Primate Neurophysiology}

An exceptional feature of the nonhuman primate brain is its similarity in development and organization to that of the human brain. A common functional architecture between monkey and human has been the foundation of significant conceptual and empirical advances in the study of cognition, no less than in the sensory (Sereno et al., 1995) and motor (Roland \& Zilles, 1996) systems of the brain. The visuospatial memory system examined in the present study is well suited to elucidate similarities or differences across phyletic order within a commonly shared functional domain-spatial memory. Two fundamental issues were addressed here. Does the same region of cortex in each primate support spatial working memory? Secondly, can similar physiological properties be demonstrated in common regions? Cytoarchitectonic maps (Rajkowska \& Goldman-Rakic, 1995) of BA $9 / 46$ (Figure 7) are very close, if not identical, to the MFG region that we now show to be strongly activated when humans represent spatial locations in short-term memory. In addition, the extended (18 or $24 \mathrm{sec}$ ) delay period used in the present study revealed fMRI signal changes in MFG that were associated with the memory component per se, with little or no contamination by responses to sensory registration and/or motor control events. These responses are analogous to the time-locked responses of prefrontal neurons to the cue, delay, and response periods of DR tasks observed in the nonhuman primate (Funahashi et al., 1989; Sawaguchi, 1987; Goldberg \& Bushnell, 1981; Kubota, Iwamoto, \& Suzuki, 1974; Niki, 1974b; Fuster \& Alexander, 1971). As in the monkey, larger amplitude activations in human cortex are associated with stimulus and response events than with the mnemonic response. Given the lower firing rates of mnemonic neural response, it is possible that such activations in human prefrontal cortex may be present but below detection with fMRI in low-demand tasks, as in tasks with only one or two memoranda (Leung, Seelig, Goldman-Rakic, \& Gore, 2000; Leung et al., 1999). The localization and profile of mnemonic activation observed here provides compelling evidence of homology between nonhuman primates and humans in the functional architecture of the dorsolateral prefrontal cortex.

\section{METHODS}

\section{Subjects and DR Tasks}

Subjects were recruited from the Yale University community, none with a history of neurological disorder according to self-report. All subjects gave informed consent to the protocol that was reviewed and approved by the Human Investigations Committee of the Yale University School of Medicine. Subjects were distributed as follows: Experiment 1: Isolation of memory component in a five-item task $(n=6$, mean age $=$ 27.7); Experiment 2: Durability of delay-related activations $(n=4$, mean age $=30.0)$; Experiment 3: Memory load effects (three items: $n=11$, mean age $=26.7$; 
five items, $n=11$, mean age $=28.2$ ). Schematic drawing for task events in each experiment is shown in Figure 1A.

\section{Experiment 1: Isolation of Memory Component in a Five-Item Task (Memory vs. No-Memory)}

Subjects were required to remember positions of five dots in the memory condition and also performed a sensorimotor task devoid of memory requirements. During each trial, five dots appeared sequentially (1 sec each) with 250-msec interstimulus intervals. After an 18-sec retention interval, a probe stimulus (1 sec) appeared, and subjects indicated whether this stimulus was at one of the memorized locations. The control task was identical except subjects were instructed not to remember anything but simply to press both buttons when the probe appeared. Each trial was $45 \mathrm{sec}$ long with a 20-sec intertrial interval (ITI). Stimulus locations ranged between $\pm 3.6^{\circ}$ and $\pm 11.5^{\circ}$ from the center. Memory or control trials were indicated by the color of the cues. Each subject performed eight runs-four runs with red/gray dots indicating the memory trials and four runs with gray/red dots indicating no-memory control trials. Thus, gray and red dots were represented equally in the memory and control conditions. The order of color and memory trial presentation was counterbalanced across runs within subjects.

\section{Experiment 2: Durability of Delay-Related Activations} (18 and $24 \mathrm{sec}$ )

Four subjects participated in additional imaging sessions. The purpose of this experiment was to determine whether the hemodynamic response could be sustained beyond the 18-sec delay interval used in the above experiments (DR-5). A total of 64 trials were collected. Half of the trials were implemented with 18-sec delays and the other half used 24-sec delays.

\section{Experiment 3: Mem ory Load Effects}

(Three vs. Five Items)

The DR-3 task in the present study is a modified version of a task employed by Courtney et al. (1999) that used pictures of faces and scrambled faces as visual stimuli. In the present version, the pictures of faces did not vary across trials and the length of delay and ITI were increased to 18 and $12 \mathrm{sec}$, respectively. Subjects were instructed to disregard the identity of the three face stimuli but to remember their locations for memory trials and not to remember anything when they saw three scrambled faces for control trials. The sequence of events in a trial is similar to that of the tasks presented above. Similarly, we also studied this task at a higher load, with five memoranda (DR-5). The total cue duration ( $6 \mathrm{sec}$ ) was equated for the two tasks. We collected seven to nine runs (four trials $\times$ two conditions per run) per subject.

\section{General Experimental Conditions}

Images were acquired with a GE 1.5 T Signa (Milwaukee, WI) scanner using the standard quadrature head coil and a T2*-sensitive gradient-recalled single-shot echo-planar pulse sequence. Axial-oblique slices $(7 \mathrm{~mm})$ were prescribed parallel to the anterior-posterior commissural (AC-PC) line. fMRI acquisition parameters were as follows: repetition time $(\mathrm{TR})=1500 \mathrm{msec}$, echo time $(\mathrm{TE})=60 \mathrm{msec}$, flip angle $=60^{\circ}$, matrix size $=64 \times 64$, and field of view $=20 \times 20 \mathrm{~cm}$. All visual stimuli were presented against a black background and back-projected onto a screen positioned at the front of the magnet bore opening. All visual stimuli were presented with PSYSCOPE software (Cohen, MacWhitney, Flatt, \& Provost, 1993) running on a Macintosh (Apple Computer, Cupertino, CA).

\section{Image Analysis-Activation and Time Courses}

A version of the SPM96 algorithm (Friston, Williams, Howard, Frackowiak, \& Turner, 1996) was used to correct for motion between successive images in each run, with images showing visible motion ( $>0.5$ pixels) removed from analysis. Images were spatially smoothed with a Gaussian filter (full width at half maximum, $6.3 \mathrm{~mm}$ ).

Activation maps for different events within a task were generated based on average percent signal changes. For each individual data set, images were aligned at the beginning of the cue of each trial and divided into blocks of four images (Figure 1B). Baseline images was formed with three before and one after cue onset. To determine task-related activations, the stimulus period (C) was defined as that between the baseline and the delay period. The 18-sec delay period was divided into three equal blocks (D1, D2, and D3), followed by the response block (R). D1 began with the second image after delay onset and $\mathrm{R}$ started from the second image after the probe onset. Images were similarly grouped for the control trials.

Individual statistical maps were transformed into a standardized coordinate system (Talairach \& Tournoux, 1988). Statistical maps of group composites were derived using a bootstrapping randomization technique (Manly, 1997). Under the null hypothesis of no signal change, the expected value for a comparison between a task event (C, D1, D2, D3, and R) and the baseline is equal to zero. The randomization creates a population distribution for each voxel by calculating multiple values for the comparison in which randomly chosen subsets of subjects' data are assigned reversed contrast weights. The randomization was performed 2000 times in order to generate an adequate sampling distribution. By comparing the experimental data to this distribution, the 
significance of each measurement can be estimated. The composite maps (Figures 2 and 3) were cluster-filtered (six contiguous pixels) and thresholded to reveal only pixel clusters with percent signal change values that fall above the 99.5th percentile of the random sampling distribution. Group composite contrast maps showing differences between the memory and control conditions of Experiments 1 and 3 were also generated using this randomization method.

ROIs were defined according to the atlas of Talairach and Tournoux (1988). Each ROI was confined to a single slice, which approximated $3-7 \mathrm{~cm}^{3}$ in volume. The average percent signal change of each ROI was calculated for each task event (C, D1, D2, D3, and R) relative to the baseline in each task. Significant signal differences between task conditions (memory and control) of both DR-3 and DR-5 tasks were tested using repeated measure analyses, with percent signal change during time blocks as the dependent variable. We focused on analyzing results from DR- 5 of Experiment 3 for 22 regions including the middle and superior frontal gyri (BA 8, 9, 46, and 10), IFG (BA 44, 45, and 47), ACG (BA 32/24), PM (BA 6), areas around the precentral sulcus (BA 6 and 44), and parietal cortex (BA 7, 40). The primary visual (BA 17/18) and motor (BA 4) areas were also reported for comparison purposes (see Table 1). In addition, differences in percent signal changes during the D3 segment of the delay interval between the control and memory conditions were assessed by paired $t$ tests. Differences were considered significant at the $p<.05$ level after Bonferroni correction for the number of comparisons.

Time course data were obtained for each ROI over the entire trial period for all tasks. Adjustments were made for variations in the time of acquisition of each slice during each TR and data were time-smoothed by a Gaussian filter (full width at half maximum, $1.5 \mathrm{sec}$ ) prior to interpolation and resampling. The average percent signal change of each ROI in a slice was calculated relative to the baseline signal for each subject. The timing of peaks was measured from the average percent signal curves of each subject, by finding the maximum value after the cue offset (first peak) and probe offset (second peak).

\section{Acknowledgments}

We thank Drs. Pawel Skudlarski and James Gatenby for their help with data analysis. We also thank Cheryl Lacadie, Terry Hickey, and Hedy Sarofin for technical assistance. This work was supported by NRSA training grant T32-NSO7416-020 and NIH grants NS33332, MH30929-22, MH38546, and MH44866.

Reprint requests should be sent to the current address of Dr. Hoi-Chung Leung, Department of Psychology, SUNY Stony Brook, Stonu Brook, NY 11794-2500, or via e-mail: hleung@ ms.cc.sunysb.edu.

The data reported in this experiment have been deposited in the fMRI Data Center (http://www.fmridc.org). The accession number is 2-2002-112P6.

\section{REFERENCES}

Baddeley, A. D. (1986). Working memory. Oxford: Clarendon Press.

Belger, A., Puce, A., Krystal, J. H., Gore, J. C., Goldman-Rakic, P., \& McCarthy, G. (1998). Dissociation of mnemonic and perceptual processes during spatial and nonspatial working memory using fMRI. Human Brain Mapping, 6, 14-32.

Carlson, S., Martinkauppi, S., Rama, P., Salli, E., Korvenoja, A., \& Aronen, H. J. (1998). Distribution of cortical activation during visuospatial $n$-back tasks as revealed by functional magnetic resonance imaging. Cerebral Cortex, 8, 743-752.

Cohen, J. D., MacWhitney, B., Flatt, M., \& Provost, J. (1993). Psyscope: A new graphic interactive environment for designing psychology experiments. Behavior Research Methods, Instruments Computers, 25, 257-271.

Cohen, J. D., Perlstein, W. M., Braver, T. S., Nystrom, L. E., Noll, D. C., Jonides, J., \& Smith, E. E. (1997). Temporal dynamics of brain activation during a working memory task [see comments]. Nature, 386, 604-608.

Courtney, S. M., Petit, L., Maisog, J. M., Ungerleider, L. G., \& Haxby, J. V. (1998). An area specialized for spatial working memory in human frontal cortex. Science, 279, 1347-1351.

D'Esposito, M., Aguirre, G. K., Zarahn, E., Ballard, D., Shin, R. K., \& Lease, J. (1998). Functional MRI studies of spatial and nonspatial working memory. Cognitive Brain Research, 7, $1-13$.

D'Esposito, M., Postle, B. R., Ballard, D., \& Lease, J. (1999). Maintenance versus manipulation of information held in working memory: An event-related fMRI study. Brain Cognition, 41, 66-86.

Duncan, J., \& Owen, A. M. (2000). Common regions of the human frontal lobe recruited by diverse cognitive demands. Trends in Neuroscience, 23, 475-483.

Friston, K. J., Williams, S., Howard, R., Frackowiak, R. S. J., \& Turner, R. (1996). Movement-related effects in fMRI time-series. Magnetic Resonance in Medicine, 35, 346-355.

Funahashi, S., Bruce, C. J., \& Goldman-Rakic, P. S. (1989). Mnemonic coding of visual space in the monkey's dorsolateral prefrontal cortex. Journal of Neurophysiology, 61, 331-349.

Fuster, J. M., \& Alexander, G. E. (1971). Neuron activity related to short-term memory. Science, 173, 652-654.

Goldberg, M. E., \& Bushnell, M. C. (1981). Behavioral enhancement of visual responses in monkey cerebral cortex: II. Modulation in frontal eye fields specifically related to saccades. Journal of Neurophysiology, 46, 773-787.

Goldberg, T. E., Berman, K. F., Randolph, C., Gold, J. M., \& Weinberger, D. R. (1996). Isolating the mnemonic component in spatial delayed response: A controlled PET ${ }^{15} \mathrm{O}$-labeled water regional cerebral blood flow study in normal humans. Neuroimage, 3, 69-78.

Goldman, P. S., Rosvold, H. E., Vest, B., \& Galkin, T. W. (1971). Analysis of the delayed-alternation deficit produced by dorsolateral prefrontal lesions in the rhesus monkey. Journal of Comparative Physiological Psychology, 77, $212-220$.

Goldman-Rakic, P. S. (1987). Circuitry of primate prefrontal cortex and regulation of behavior by representational memory. In V. B. Mountcastle \& F. Plum (Eds.), Handbook of physiology: The nervous system, higher functions of the brain (vol. 5, pp. 373-417). Bethesda, MD: American Physiological Society.

Goldman-Rakic, P. S. (1996). The prefrontal landscape: Implications of functional architecture for understanding human mentation and the central executive. Philosophical Transactions of the Royal Society of London, Series B: Biological Sciences, 351, 1443-1453. 
Jiang, Y., Olson, I. R., \& Chun, M. M. (2000). Organization of visual short-term memory. Jou rnal of Experimental Psychology, Learning, Memory, and Cognition, 26, $683-702$.

Jonides, J., Smith, E. E., Koeppe, R. A., Awh, E., Minoshima, S., \& Mintun, M. A. (1993). Spatial working memory in humans as revealed by PET [see comments]. Nature, 363, 623-625.

Kubota, K., Iwamoto, T., \& Suzuki, H. (1974). Visuokinetic activities of primate prefrontal neurons during delayedresponse performance. Journal of Neurophysiology, 37, $1197-1212$.

Leung, H.-C., Gore, J. C., \& Goldman-Rakic, P. S. (1999). Dissociation of cue, delay and response activity during a spatial working memory task by event-related fMRI. Society for Neuroscience Abstracts, 25, 44.3.

Leung, H.-C., Seelig, D., Goldman-Rakic, P. S., \& Gore, J. C. (2000). Effect of spatial working memory load on brain activation using event-related fMRI. Society for Neu roscience Abstracts, 26, 560.15 .

Levy, R., \& Goldman-Rakic, P. S. (1999). Association of storage and processing functions in the dorsolateral prefrontal cortex of the nonhuman primate. Journal of Neuroscience, 19, $5149-5158$.

Manly, B. F. J. (1997). Randomization, bootstrap, and Monte Carlo methods in biology, 2nd ed. London: Chapman \& Hall.

McCarthy, G., Puce, A., Constable, R. T., Krystal, J. H., Gore, J. C., \& Goldman-Rakic, P. (1996). Activation of human prefrontal cortex during spatial and nonspatial working memory tasks measured by functional MRI. Cerebral Cortex, 6, 600-611.

Miyake, A., \& Shah, P. (1999). Models of working memory: Mechanisms of active maintenance and executive control. Cambridge: Cambridge University Press.

Niki, H. (1974a). Differential activity of prefrontal units during right and left delayed response trials. Brain Research, 70, $346-349$.

Niki, H. (1974b). Prefrontal unit activity during delayed alternation in the monkey: I. Relation to direction of response. Brain Research, 68.

O'Sullivan, E. P., Jenkins, I. H., Henderson, L., Kennard, C., \& Brooks, D. J. (1995). The functional anatomy of remembered saccades: A PET study. NeuroReport, 6, 2141-2144.

Owen, A. M. (1997). The functional organization of working memory processes within human lateral frontal cortex: The contribution of functional neuroimaging. European Journal of Neu roscience, 9, 1329-1339.

Owen, A. M., Evans, A. C., \& Petrides, M. (1996). Evidence for a two-stage model of spatial working memory processing within the lateral frontal cortex: A positron emission omography study. Cerebral Cortex, 6, 31-38.

Owen, A. M., Herrod, N. J., Menon, D. K., Clark, J. C., Downey, S. P., Carpenter, T. A., Minhas, P. S., Turkheimer, F. E., Williams, E. J., Robbins, T. W., Sahakian, B. J., Petrides, M., \& Pickard, J. D. (1999). Redefining the functional organization of working memory processes within human lateral prefrontal cortex. European Journal of Neuroscience, 11, $567-574$.

Paus, T. (1996). Location and function of the human frontal eye-field: A selective review. Neuropsychologia, 34, $475-483$.

Petrides, M. (1994a). Frontal lobes and behaviour. Current Opinions in Neurobiology, 4, 207-211.
Petrides, M. (1994b). Frontal lobes and working memory: Evidence from investigations of the effects of cortical excisions in nonhuman primates. In F. Boller \& J. Grafman (Eds.), Handbook of neuropsychology (vol. 9, pp. 59-82). Amsterdam: Elsevier.

Postle, B. R., Berger, J. S., \& D’Esposito, M. (1999). Functional neuroanatomical double dissociation of mnemonic and executive control processes contributing to working memory performance. Proceedings of the National Academy of Sciences, U.S.A., 96, 12959-12964.

Rajkowska, G., \& Goldman-Rakic, P. S. (1995). Cytoarchitectonic definition of prefrontal areas in the normal human cortex: II. Variability in locations of areas 9 and 46 and relationship to the Talairach coordinate system. Cerebral Cortex, 5, 323-337.

Roland, P. E., \& Zilles, K. (1996). Functions and structures of the motor cortices in humans. Current Opinions in Neurobiology, 6, 773-781.

Rowe, J. B., Toni, I., Josephs, O., Frackowiak, R. S., \& Passingham, R. E. (2000). The prefrontal cortex: Response selection or maintenance within working memory? Science, 288, 1656-1660.

Rypma, B., Prabhakaran, V., Desmond, J. E., Glover, G. H., \& Gabrieli, J. D. (1999). Load-dependent roles of frontal brain regions in the maintenance of working memory. Neuroimage, 9, 216-226.

Sawaguchi, T. (1987). Properties of neuronal activity related to a visual reaction time task in the monkey prefrontal cortex. Journal of Neurophysiology, 58, 1080-1099.

Sereno, M. I., Dale, A. M., Reppas, J. B., Kwong, K. K., Belliveau, J. W., Brady, T. J., Rosen, B. R., \& Tootell, R. B. (1995). Borders of multiple visual areas in humans revealed by functional magnetic resonance imaging [see comments]. Science, 268, 889-893.

Smith, E. E., \& Jonides, J. (1999). Storage and executive processes in the frontal lobes. Science, 283, 1657-1661.

Smith, E. E., Jonides, J., \& Koeppe, R. A. (1996). Dissociating verbal and spatial working memory using PET [published erratum appears in Cereb Cortex 1998 Dec;8(8):262] . Cerebral Cortex, 6, 11-20.

Smith, E. E., Jonides, J., Koeppe, R. A., Awh, E., Schumacher, E. H., \& Minoshima, S. (1995). Spatial versus object working memory: PET investigations. Journal of Cognitive Neuroscience, 7, 337-356.

Smyth, M. M., \& Scholey, K. A. (1992). Determining spatial memory span: The role of movement time and articulation rate. Quarterly Journal of Experimental Psychology, 45A, 479-501.

Sweeney, J. A., Mintun, M. A., Kwee, S., Wiseman, M. B., Brown, D. L., Rosenberg, D. R., \& Carl, J. R. (1996). Positron emission tomography study of voluntary saccadic eye movements and spatial working memory. Journal of Neu rophysiology, 75, 454-468.

Talairach, J., \& Tournoux, P. (1988). Co-planar stereotaxic atlas of the human brain. New York: Thieme.

Zarahn, E., Aguirre, G. K., \& D'Esposito, M. (1999). Temporal isolation of the neural correlates of spatial mnemonic processing with fMRI. Cognitive Brain Research, 7, 255-268.

Zarahn, E., Aguirre, G. K., \& D'Esposito, M. (2000). Replication and further studies of neural mechanisms of spatial mnemonic processing in humans. Brain Research Cognitive Brain Research, 9, 1-17. 